\title{
A lattice Boltzmann study of dynamic immiscible displacement mechanisms in pore doublets
}

\author{
Mohammad Sadeghi ${ }^{1}$, Hamed Sadeghi ${ }^{1, *}$, and Clarence E. Choi ${ }^{2}$ \\ ${ }^{1}$ Sharif University of Technology, Department of Civil Engineering, Tehran, Iran \\ ${ }^{2}$ The University of Hong Kong, Department of Civil Engineering, Hong Kong
}

\begin{abstract}
An advanced chromodynamics, Rothmann-Keller (RK) type lattice Boltzmann model (LBM) is used in this study. The new model benefits from high stability and capability of independently setting the interfacial tension of the fluids as an input parameter. In addition, the model is coupled with a wall-density approach to simulate the hydrophilic or hydrophobic properties of wall surfaces. Finally, injection of a wetting (non-wetting) fluid in a pore doublet geometry which is initially filled with non-wetting (wetting) fluid is simulated. The results of simulation reveal the capability of RK-LBM to simulate relative permeabilities of fluids in porous media for future studies of two-immiscible phase flow in various geoenvironmental problems.
\end{abstract}

\section{Introduction}

Pore scale study of multiphase flow through porous media finds application in areas such as flow of water in vadose zone Sadeghi et al., (2020) [1], transport of pollution in soil, oil recovery, and hydrofracturing for natural gas production. Therefore, extensive laboratory studies have been carried out to explore the fundamental mechanisms governing the single-phase flow Sadeghi \& AliPanahi, (2020) [2] and two-phase flow-dependent characteristics of porous media Bazargan et al., (2015) [3]; Sadeghi \& Nasiri, (2020) [4]. More importantly, numerical investigations have always been in the research front because of their robust, yet flexible modelling capabilities Kolahdooz et al., (2020) [5]. For example, Ghassemi \& Pak (2011a) [6] studied factors influencing relative permeabilities of two immiscible fluids flowing through porous media numerically using lattice Boltzmann method (LBM). Ghassemi \& Pak (2011b) [7] also studied permeability and tortuosity of flow in porous media using (LBM).

Three types of popular lattice Boltzmann models are widely used to study multiphase flow regimes. These models are chromodynamics (RK) model proposed by Rothman \& Keller (1988) [8], and Gunstensen et al. (1991) [9]; pseudopotential (SC) model introduced by Shan \& Chen (1993) [10]; and free-energy (FE) method of Swift et al. (1996) [11]. The RK model developed by Leclaire et al. (2011 [12], 2012 [13]) has several benefits over others, including flexible parameter adjustment, wide range of input parameters, high stability, strict mass conservation, and exact modelling of interfacial tension. This model coupled with wall density approach of LatvaKoko \& Rothman (2005) [14] to simulate hydrophobic or hydrophilic wall surfaces. Therefore, it is used in this study to simulate and explore the immiscible displacement in a pore doublet.

\section{Lattice Boltzmann method (LBM)}

The RK-LBM described here was originally developed by Leclaire et al. (2011 [12], 2012 [13]). The discretized form of Boltzmann equation which is numerically solved is given in Eq. (1) where $N_{i}^{k}(\boldsymbol{x}, t)$ represents the distribution function for a fluid of color $k$ in $i$ direction, $\boldsymbol{x}$ is the node position, $t$ is time step, and $\Omega_{i}^{k}$ is the collision operator, which itself consists of three other sub-operators including 1 single-phase operator and 2 two-phase operators.

$$
N_{i}^{k}\left(\boldsymbol{x}+\boldsymbol{c}_{i}, t+1\right)=N_{i}^{k}(\boldsymbol{x}, t)+\Omega_{i}^{k}(\boldsymbol{x}, t)
$$

\subsection{Streaming operator}

The RK-LBM implementation consists of six parts in each time step. At the beginning of each time step, a singlephase streaming operator is responsible for linear streaming of the distribution functions of each fluid in a standard D2Q9 lattice with the nine predefined velocity vectors $c_{i}$. Velocity vectors $\boldsymbol{c}_{i}$ for all nine directions are given in Eq. (2).

$$
\boldsymbol{c}_{i}=\left\{\begin{array}{lr}
(0,0), & i=1 \\
{\left[\sin \left(\theta_{i}\right), \cos \left(\theta_{i}\right)\right],} & i=2,4,6,8 \\
{\left[\sin \left(\theta_{i}\right), \cos \left(\theta_{i}\right)\right] \sqrt{2},} & i=3,5,7,9
\end{array}\right.
$$

And the all nine values of $\theta_{i}$ in Eq. (2) are calculated according to Eq. (3)

$$
\theta_{i}=(4-i)(\pi / 4)
$$

\footnotetext{
* Corresponding author: hsadeghi@sharif.edu
} 
Then the macroscopic quantities such as fluid densities $\rho_{k}$, and fluid velocity $\boldsymbol{u}$ are obtained from Eq. (4) and Eq. (5), respectively.

$$
\begin{gathered}
\rho_{k}=\sum_{i} N_{i}^{k} \\
\boldsymbol{u}=\left(\sum_{i} \sum_{k} N_{i}^{k} \boldsymbol{c}_{i}\right) / \sum_{\boldsymbol{k}} \rho_{k}
\end{gathered}
$$

\subsection{Boundary conditions}

Based on the calculated macroscopic quantities, in the second part of each time step, all boundary conditions are implemented. They include one-directional and fully periodic boundaries, simple bounceback boundary, and Von Neumann (Flux) boundaries. The implementation of boundaries is similar to the work of Sukop \& Thorne (2006) [15]. For implementation of Dirichlet (Pressure) boundaries, the more accurate method proposed by Machado (2012) [16] is used.

\subsection{Single-phase collision operator}

The third part of each time step is a single-phase collision operator $\Omega_{i}^{k(I)}$, which is calculated from Eq. (6). This operator is the standard BGK or single-relaxation-time (SRT) approximation, in which the distribution functions are relaxed toward a local equilibrium. The $\omega_{k}$ is the relaxation factor of fluid $k$.

$$
\left(\Omega_{i}^{k}\right)^{(1)} N_{i}^{k}=N_{i}^{k}-\omega_{k}\left(N_{i}^{k}-N_{i}^{k(e)}\right)
$$

The equilibrium distribution functions $N_{i}^{k(e)}$ given in Eq. (7) are chosen to take into account the principles of mass and momentum conservation.

$$
N_{i}^{k(e)}=\rho_{k}\left(\phi_{i}^{k}+W_{i}\left[3 \boldsymbol{c}_{i} \cdot \boldsymbol{u}+\frac{9}{2}\left(\boldsymbol{c}_{i} \cdot \boldsymbol{u}\right)^{2}-\frac{3}{2} \boldsymbol{u}^{2}\right]\right)(7)
$$

The weights $W_{i}$ are those of a standard D2Q9 lattice. $\phi_{i}$ values are given in Table 1, and the arbitrary parameters of $\alpha_{k}$ are chosen to satisfy Eq. (8).

$$
\rho_{r} / \rho_{b}=\left(1-\alpha_{b}\right) /\left(1-\alpha_{r}\right)
$$

Table 1. D2Q9 parameters: $W_{i}, \phi_{i}^{k}, B_{i}$.

\begin{tabular}{cccc}
\hline$i$ & $W_{i}$ & $\phi_{i}^{k}$ & $B_{i}$ \\
\hline 1 & $4 / 9$ & $\alpha_{k}$ & $-4 / 27$ \\
$2,4,6,8$ & $1 / 9$ & $\left(1-\alpha_{k}\right) / 5$ & $+2 / 27$ \\
$3,5,7,9$ & $1 / 36$ & $\left(1-\alpha_{k}\right) / 20$ & $+5 / 108$ \\
\hline
\end{tabular}

The fluid viscosity $v_{k}$ determines the relaxation parameter $\omega_{k}$ through Eq. (9). Similar to the work of Leclaire et al. (2011) [12], for different fluid viscosities, a type of interpolation is required to define the parameter $\omega$ at the interface. To do this, a color field parameter $\psi$ was introduced which maps between -1 and 1 . At an interface, the relaxation factor $\omega_{k}$ is replaced by $\omega$ from Eq. (10).

$$
\begin{gathered}
\omega_{k}=1 /\left(3 v_{k}+0.5\right) \\
\omega=\left\{\begin{array}{lr}
\omega_{r}, & \psi>\delta \\
f_{r}(\psi), & \delta \geq \psi>0 \\
f_{b}(\psi), & 0 \geq \psi \geq-\delta \\
\omega_{b} & \psi<-\delta
\end{array}\right.
\end{gathered}
$$

In Eq. (10), $\delta$ is a free parameter which affects the thickness of the interface. The color field functions are defined in Eq. (11), and Eq. (12).

$$
\begin{aligned}
& f_{r}(\psi)=\chi+\eta \psi+\kappa \psi^{2} \\
& f_{b}(\psi)=\chi+\lambda \psi+v \psi^{2}
\end{aligned}
$$

The required parameters used in Eq. (11) and Eq. (12) are obtained from equations 13 to 17 .

$$
\begin{gathered}
\chi=2 \omega_{r} \omega_{b} /\left(\omega_{r}+\omega_{b}\right) \\
\eta=2\left(\omega_{r}-\chi\right) / \delta \\
\kappa=-\eta / 2 \delta \\
\lambda=2\left(\chi-\omega_{b}\right) / \delta \\
v=\lambda / 2 \delta
\end{gathered}
$$

\subsection{Color gradient vector calculation}

\subsubsection{Sixth order isotropic color gradient}

The fourth part of each time step is the calculation of color gradient $\mathbf{F}$, which is a vector perpendicular to the interface between fluids, points toward the red region. Color gradient vector is required for next two steps, namely perturbation and recoloring. To calculate the sixth order isotropic color gradient vector $\mathbf{F}$ for each node of the lattice, the two outer layer nodes ( 24 nodes) are required. The sixth order isotropic color gradient is gained by Eq. (18).

$$
\boldsymbol{F}(\boldsymbol{x})=\sum_{i=1}^{i=25} \xi_{i} \boldsymbol{d}_{i} \Delta\left(\boldsymbol{x}+\boldsymbol{d}_{i}\right)
$$

The twenty-five weight components $\xi_{i}$, and the horizontal $\boldsymbol{d}_{x}$ and vertical $\boldsymbol{d}_{y}$ components of each of twenty-five vectors $\boldsymbol{d}_{i}$ are defined in Eq. $(19,20,21)$ respectively.

$$
\begin{gathered}
\xi=\frac{[0,960,488,960,488,960,488,960,488,84,32,1,32,84,32,1,32,84,32,1,32,84,32,1,32]}{5040} \\
\boldsymbol{d}_{x}=[0,0,1,1,1,0,-1,-1,-1,0,1,2,2,2,2,2,1,0,-1,-2,-2,-2,-2,-2,-1] \\
\boldsymbol{d}_{y}=[0,1,1,0,-1,-1,-1,0,1,2,2,2,1,0,-1,-2,-2,-2,-2,-2,-1,0,1,2,2]
\end{gathered}
$$

\subsubsection{Hydrophilic / hydrophobic wall surface}

In case of calculating color gradient for fluid nodes near the solid nodes (within two outer layers), some fake densities of either red or blue fluids were assigned to solid nodes. These fake densities won't flow through the fluid 
domain, and are only used in this step to change the magnitude and direction of the color gradient vector $\mathbf{F}$. Sadeghi et al. (2019) [17] introduced a red percentage parameter $P_{\text {red }}$ so that the $P_{\text {red }}$ portion of the initial red fluid density is poured into solid nodes. It was shown that the wettability of the surface and even the contact angles of fluids with wall surface is adjustable through the red percentage parameter. The red percentage parameter takes a value between -1 and 1 . If it sets to 1 , the red fluid is completely wetting with contact angle of $0^{\circ}$ near wall, and if it sets to -1 , the red fluid is completely non-wetting with contact angle of $180^{\circ}$ near wall. Latva-Koko and Rothman (2005) [14] proved that for two immiscible fluids of same density and viscosity in RK-LBM, the contact angle $\theta$ of the red fluid with surface can be obtained from Eq. (22).

$$
\theta=\operatorname{Arccos}\left(P_{\text {red }}\right)
$$

Sadeghi et al. (2019) [17] recommended to perform an obligatory capillary rise simulation for non-identical fluids, and then calculate the contact angle $\theta$ from Eq. (23). In the following equation, $\theta$ is contact angle, $\rho$ is density, $g$ is ground acceleration, $w$ is the width of tube, $\sigma$ is surface tension, and $h$ is capillary height obtained from simulation for the specific $P_{\text {red }}$ parameter.

$$
\theta=\operatorname{Arccos}(\rho g h w / 2 \sigma)
$$

\subsection{Two-phase perturbation operator}

The fifth part of each loop is the first two-phase collision operator, called perturbation operator. This operator is responsible for modelling surface tension between fluids. While this operator accurately models the surface tension between fluids, it does not satisfy the immiscibility of fluids. The perturbation operator $\Omega_{i}^{k(2)}$ is calculated according to Eq. (24). According to the work of Reis and Phillips (2007) [18], this operator follows the macroscopic equations for two-phase fluid flow. The values for $B_{i}$ are given in table 1 .

$$
\left(\Omega_{i}^{k}\right)^{(2)} N_{i}^{k}=N_{i}^{k}+\frac{A_{k}}{2}|\boldsymbol{F}|\left[W_{i} \frac{\left(\boldsymbol{F} \cdot \boldsymbol{c}_{\boldsymbol{i}}\right)^{2}}{|\boldsymbol{F}|^{2}}-B_{i}\right]
$$

In Eq. (24), $A_{k}$ is a free parameter which is chosen to adjust the surface tension according to Eq. (25). Sadeghi (2013) [19] studied the stability and accuracy of the surface tension parameter of the model for two fluids with densities and viscosities similar to water and air. The bubble test simulation results were validated against the well-known Laplace's law with excellent match.

$$
\sigma=\left(\rho_{r}+\rho_{b}\right)\left(A_{r}+A_{b}\right) / 9 \omega
$$

\subsection{Two-phase recoloring operator}

As mentioned earlier, to guarantee the immiscibility of fluids, a recoloring operator is required which is the sixth and last part of each time step. The operator's duty is to send the maximum amount of each color fluid (red or blue) at the interface, to the bulk region of that specific color fluid (red or blue). The recoloring operator $\Omega_{i}{ }^{k(3)}$ for both fluids is presented in Eq. $(26,27)$.

$$
\begin{aligned}
& \left(\Omega_{i}^{r}\right)^{(3)} N_{i}^{r}=\frac{\rho_{r}}{\rho} N_{i}+\beta \frac{\rho_{r} \rho_{b}}{\rho^{2}} \cos \varphi_{i} \sum_{k} N_{i}^{k(e)}\left(\rho_{k}, \boldsymbol{u}_{0}\right) \\
& \left(\Omega_{i}^{b}\right)^{(3)} N_{i}^{b}=\frac{\rho_{b}}{\rho} N_{i}-\beta \frac{\rho_{r} \rho_{b}}{\rho^{2}} \cos \varphi_{i} \sum_{k} N_{i}^{k(e)}\left(\rho_{k}, \boldsymbol{u}_{0}\right)
\end{aligned}
$$

In previous equations $\beta$ is a free parameter between 0 and 1 , which controls the thickness of the interface. The more its value, the thinner the interface is. And $\varphi_{i}$ is the angle between color gradient $\mathbf{F}$, and the direction $c_{i}$ in a D2Q9 lattice. The equilibrium distribution functions for each fluid $N_{i}^{k(e)}$ in Eqs (26) and (27) are evaluated with a zero velocity $\boldsymbol{u}_{0}=\boldsymbol{u}=0$.

\section{Poiseuille flow simulation}

Poiseuille flow is fluid flow between two parallel plates, which is caused by applying pressure difference $\Delta p$ at inlet and outlet of the canal or by applying an acceleration along the canal (parallel to the plates).

\subsection{Single-phase Poiseuille flow}

Figure 1 shows the schematic domain. The analytical solution for the velocity profile of the Poiseuille flow is given in equation 28 .

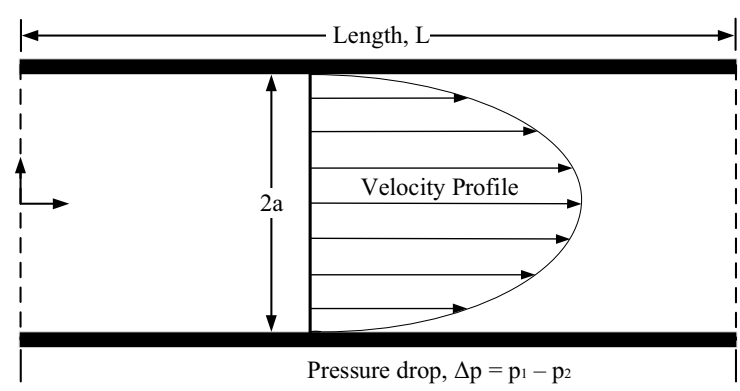

Fig. 1. Schematic of Poiseuille flow domain between two plates.

$$
u(y)=\frac{\Delta p \cdot a^{2}}{2 \mu \cdot L}\left[1-\left(\frac{y}{a}\right)^{2}\right]
$$

Where $y$ is the vertical position from the middle line in canal, $u$ is the velocity, $\Delta p$ is pressure difference at the inlet and outlet, $a$ is half of the width of the canal, $\mu$ is dynamic viscosity of fluid, $L$ is the length of canal. The LBM simulation is done in a $62 \times 300$ canal. The simulation results, and analytical solution are plotted in Figure 2. The comparison of results suggests the high accuracy of LBM in capturing this phenomenon. 


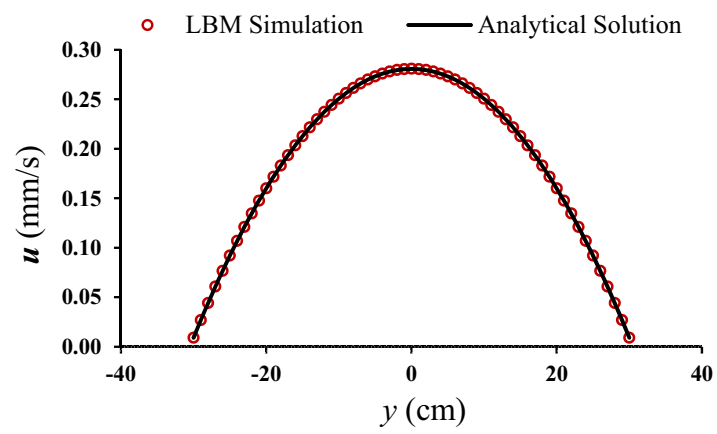

Fig. 2. Poiseuille flow: LBM simulation results (points) compared to analytical solution (line).

\subsection{Two-phase Poiseuille flow}

A two-phase Poiseuille flow is simulated between two parallel plates, where the $25 \%$ of the width of the canal in each side is filled with wetting fluid, and the remaining $50 \%$ percent in the middle is filled with non-wetting fluid. Figure 3 depicts the initial state, and boundary conditions implemented in the simulation.

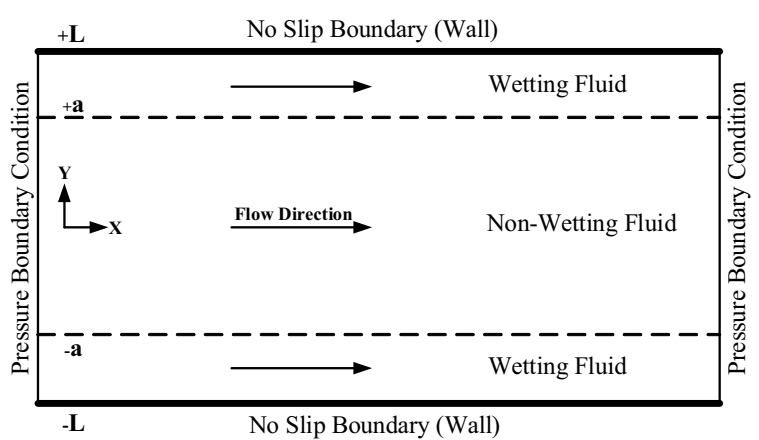

Fig. 3. Schematic of two-phase Poiseuille flow domain between two plates: Initial state and boundary conditions.

The analytical solution for the problem is given for wetting fluid, and non-wetting fluid in Eqs (29) and (30), respectively; where $\boldsymbol{u}$ is the macroscopic velocity in vertical position $y$, and $\Delta p$ is the pressure difference along the length of canal $l$. The dynamic viscosities for wetting and non-wetting fluids are $\mu_{w}, \mu_{n w}$. In addition, $L, a$ are shown in the schematic Figure 3.

$$
\begin{gathered}
\boldsymbol{u}_{w}(y)=\frac{\Delta p}{2 l \mu_{w}}\left(L^{2}-y^{2}\right) \\
\boldsymbol{u}_{n w}(y)=\frac{\Delta p}{2 l \mu_{w}}\left(L^{2}-a^{2}\right)+\frac{\Delta p}{2 l \mu_{n w}}\left(a^{2}-y^{2}\right)
\end{gathered}
$$

Two types of simulation are done, with the viscosity ratios of 10 and 0.1 , and also different pressure differences. The results for velocity profile of each of them is plotted against the analytical solution in figures 4, 5; which shows the capability, and accuracy of LBM for simulating two-phase flows in a canal. When $M$ which is defined as the viscosity ratio of non-wetting fluid $v_{n w}$ to the wetting one $v_{w}$; is more than 1 , the middle fluid flows more freely and speeds up more than the side one, but when the middle fluid is more viscose, it could not flow as freely as the wetting fluid.

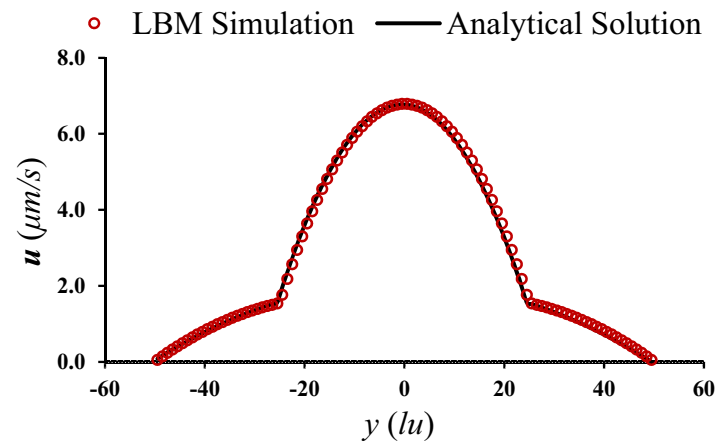

Fig. 4. Two-phase Poiseuille flow: LBM simulation and analytical solution for $M=v_{n w} / v_{w}=10$.

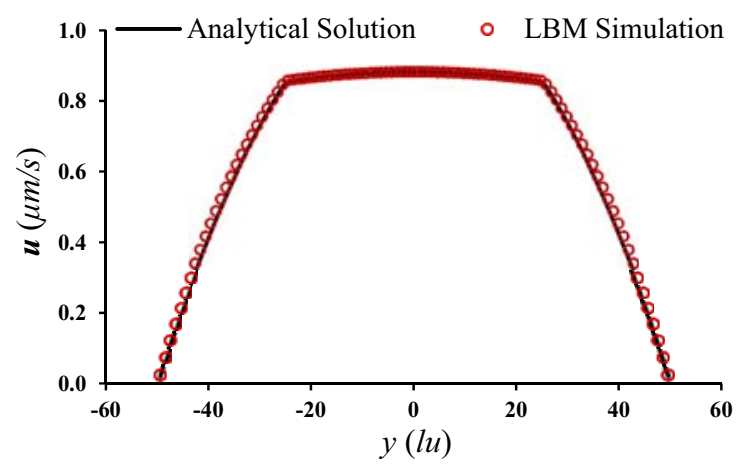

Fig. 5. Two-phase Poiseuille flow: LBM simulation and analytical solution for $=v_{n w} / v_{w}=0.1$.

\section{Dynamic immiscible displacement in pore doublets}

The geometry of a pore doublet model consists of two canals with non-identical widths, which laid in one wider canal. Due to inverse proportionality of capillary pressure to the width of a canal; the capillary pressure of the narrower canal is greater than that of the wider one. A flux (Von Neumann) boundary condition applied at the left side or inlet of the canal depicted in Figure 6, is responsible for injecting wetting (non-wetting) fluid to the domain which has been already filled with non-wetting (wetting) fluid. The pressure (Dirichlet) boundary condition applied at the right side or outlet of the canal, drain the fluid from domain as the injecting fluid comes in.

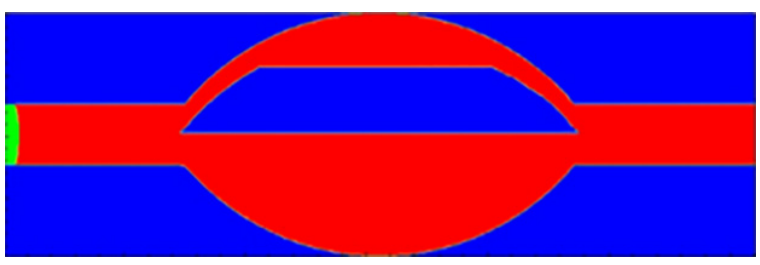

Fig. 6. Initial state of drainage-type snap-off in a doublet. 
The pressure gradient between outlet and inlet should be adjusted to be greater than the capillary pressure of the wider canal, but simultaneously less than that of the narrower canal. This causes the non-wetting fluid not to enter the narrow canal, and the wetting fluid to be trapped inside the narrow canal when the non-wetting fluid reaches the outlet. Lenormand et al. (1983) [20] and, Pak \& Sheikh (2013) [21] showed this phenomenon experimentally and theoretically, respectively. Figure 7 shows the injection of wetting fluid (a), and non-wetting fluid (b) to the canal. The injection speed was too low, and a creeping regime governed the flow, so the curvature of the interface between fluids is large. Because of the hydrophilic wall surface, the wetting fluid in Figure (a) preferably flow to the narrow canal, and it creeped on the surface of the wall almost to the end of canal. It is obvious that the non-wetting fluid did not enter the narrow canal, and the curvature is opposite of what we saw in injection of wetting fluid. Figure 7 shows the result of simulaton at the end of twenty-million time steps, which took almost two months on a single CPU core. Beside the fact that the injection speed at the inlet was too low, LBM is computationally demanding and this long run duration is Achilles' heel of our C++ code which has not yet parallelized. To cope with this high computational demand, Montellà et al, (2020) [22] proposed a hybrid Method and coupled LBM with pore-network model. Obrecht et al. (2013) obtained excellent performance for LB simulations on GPU clusters using the CUDA code.
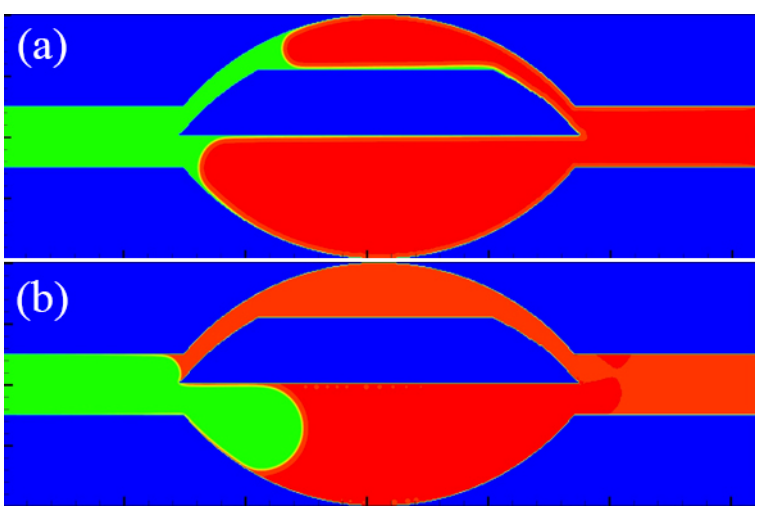

Fig. 7. Drainage-type snap-off in a doublet at final state (a) for wetting fluid injection, and (b) for non-wetting fluid injection.

As reaching of the injecting fluid to the outlet was not seen in previous simulation, the injection speed at the inlet increased to see it. This simulation took just several days, but the pressure gradient was higher than the capillary pressure in both canals and the injected non-wetting fluid in Figure 8(b) preferably entered the wider canal, but finally has entered the narrow one. Only small amount of wetting fluid trapped near the right-side wall of the canals. Figure 8(a) shows the evolution of flow at the same time steps as in Figure 8(b). The injected wetting fluid wiped out total amount of non-wetting fluid to the out of domain. The curvature of interface between two fluids is lower than those of previous simulations depicted in figure 7.

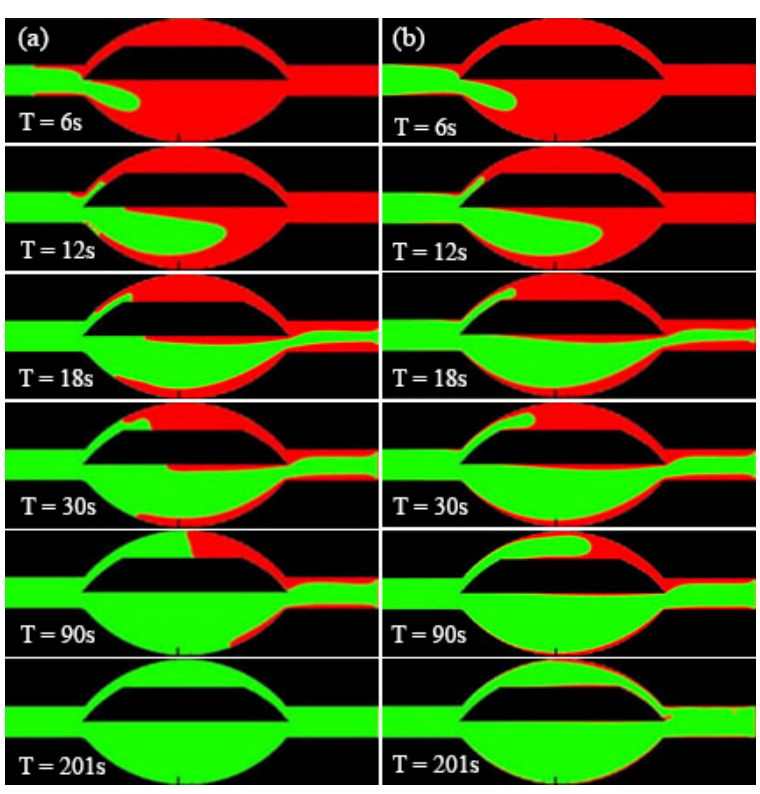

Fig. 8. Drainage-type snap-off evolution in a doublet at six specific time steps: (a) injection of wetting fluid, and (b) injection of non-wetting fluid.

\section{Conclusions}

A stable Rothmann-Keller type lattice Boltzmann method RKLBM was implemented in $\mathrm{C}++$ programming language. The code was validated using two benchmark single-phase, and two-phase Poiseuille flow. Afterwards, a drainage-type snap-off in pore doublets was simulated. The solid phase in the domain was set to be fully hydrophilic or fully hydrophobic by means of the $P_{\text {red }}$ parameter previously introduced by Sadeghi et al (2019). In addition to these fundamental phenomena studied here, further developments of the stability proven code including its parallelisation, are under way for simplifying simulation of more complex flow phenomena such as relative permeability. However, current simulations in such a simple two-dimensional geometry could not be a representative for flow of fluids in a real threedimensional complex porous media. For future work, we will study relative permeabilities of two-phase fluid flow in porous media using the newly developed RKLBM code.

\section{References}

1. H. Sadeghi, C.F. Chiu, C.W.W. Ng, F. Jafarzadeh. (2020). A vacuum-refilled tensiometer for deep monitoring of in-situ pore water pressure. Scientia Iranica. $\quad 27: 2, \quad 596-606, \quad$ doi: 10.24200/sci.2018.5052.1063

2. H. Sadeghi, \& P. AliPanahi. (2020). Saturated hydraulic conductivity of problematic soils measured by a newly developed low-compliance triaxial permeameter. Eng. Geol., 278, 105827. doi: 10.1016/j.enggeo.2020.105827

3. A. Bazargan, H. Sadeghi, R. Garcia-Mayoral, G. McKay. (2015). An unsteady state retention model 
for fluid desorption from sorbents. J. Colloid Interface Sci., 450, 127-134, doi: 10.1016/j.jcis.2015.02.036

4. H. Sadeghi, \& H. Nasiri. (2020). Hysteresis of soil water retention and shrinkage behaviour for various salt concentrations. Géotechnique Letters. 11:1, 2129, doi: 10.1680/jgele.20.00047.

5. A. Kolahdooz, H. Sadeghi,M.M. Ahmadi. (2020). A numerical study on the effect of salinity on stability of an unsaturated railway embankment under rainfall. 4th European Conference on Unsaturated Soils (EUNSAT 2020), 195 (01004), 1-6, doi: 10.1051/e3sconf/202019501004

6. A. Ghassemi \& A. Pak. (2011). Numerical Study of Factors Influencing Relative Permeabilities of Two Immiscible Fluids Flowing through Porous Media using Lattice Boltzman Method. J. Petrol Sci. Eng., 77, 135-145, doi: 10.1016/j.petrol.2011.02.007

7. A. Ghassemi\& A. Pak. (2011). Pore Scale Study of Permeability and Tortuosity for Flow through Particulate Media Using Lattice Boltzman Method. Int. J. Numer. Anal. Methods Geomech., 35, 886901, doi: 10.1002/nag.932

8. D.H. Rothman \& J.M. Keller. (1988). Immiscible cellular-automaton fluids. J. Stat. Phys., 52 :3-4, 1119-1127, doi: 10.1007/BF01019743

9. A.K. Gunstensen, D.H. Rothman, S. Zaleski,G. Zanetti. (1991). Lattice Boltzmann model of immiscible fluids. Phys. Rev. A, 43 :8, 4320-4327, doi: 10.1103/PhysRevA.43.4320

10. X. Shan \& H. Chen. (1993). Lattice Boltzmann model for simulating flows with multiple phases and components. Phys. Rev. E, 47 :3, 1815-1819, doi: 10.1103/PhysRevE.47.1815

11. M.R. Swift, E. Orlandini,W.R. Osborn,J.M. Yeomans. (1996). Lattice Boltzmann simulation of liquid-gas and binary fluid systems. Phys. Rev. E, 54 :5, 5041-5052, doi: 10.1103/PhysRevE.54.5041

12. S. Leclaire,M. Reggio,J.Y. Trepanier. (2011). Isotropic color gradient for simulating very highdensity ratios with a two-phase flow lattice Boltzmann model. Comput. Fluids, 48 :1, 98-112, doi: 10.1016/j.compfluid.2011.04.001

13. S. Leclaire, M. Reggio, J.Y. Trepanier. (2012). Numerical Evaluation of two recoloring operators for an immiscible two-phase flow lattice Boltzmann model. Appl. Math. Model., 36, 2237-2252, doi: 10.1016/j.apm.2011.08.027

14. M. Latva-Koko \&D.H. Rothman. (2005). Static contact angle in lattice Boltzmann models of immiscible fluids. Phys. Rev. E, 72 :4, 046701, doi: 10.1103/PhysRevE.72.046701

15. M.C. Sukop \& D.T. Thorne. (2006). Lattice Boltzmann Modeling: An Introduction for geoscientists and engineers (Springer, Heidelberg, Berlin, New York)

16. R. Machado. (2012). On pressure and corner boundary conditions with two lattice Boltzmann construction approaches. Math. Comput. Simul., 84, 26-41, doi: 10.1016/j.matcom.2012.08.002

17. M Sadeghi, A. Pak, H. Sadeghi. (2019). Simulation of wetting tendency of fluids with high density ratios using RK Lattice Boltzmann method. The 16th Asian Regional Conference on Soil Mechanics and Geotechnical Engineering (16ARC), Oct. 2019, Taipei, Taiwan.

18. T. Reis \& T.N. Phillips. (2007). Lattice Boltzmann model for simulating immiscible two-phase flows. J. Phys. A Math. Theor., 40:14, 1151-1173, doi: 10.1088/1751-8113/40/14/018

19. M. Sadeghi. (2013). Evaluation of efficiency of RK chromodynamic model for simulation of two-fluid flow in porous media. MSc Thesis, Sharif University of Technology.

20. R. Lenormand, C. Zarcone, A. Sarr. (1983). Mechanisms of the displacement of one fluid by another in a network of capillary ducts. J. Fluid Mech., 135, 337-353, doi: 10.1017/S0022112083003110

21. A. Pak \& B. Sheikh. (2013) Study of Relative Permeability Variation During Unsteady Flow in Unsaturated Reservoir Rock Using Lattice Boltzmann Method. Proc. 18th International Conference on Soil Mechanics and Geotechnical Engineering, Paris.

22. E.P. Montellà, C. Yuan, B. Chareyre, A. Gens. (2020). Modeling multiphase flow with a hybrid model based on the Pore-network and the lattice Boltzmann method. 4th European Conference on Unsaturated Soils (E-UNSAT 2020), 195, (02009),doi: 10.1051/e3sconf/202019502009

23. C. Obrecht, F. Kuznik, B. Tourancheau, J. Roux. (2013). Scalable lattice Boltzmann solvers for CUDA GPU clusters. Parallel Computing., 39 :6-7, 259-270, doi: 10.1016/j.parco.2013.04.001 